\title{
CoLiTec software - detection of the near-zero apparent motion
}

\author{
Sergii V. Khlamov ${ }^{1} \dagger$, Vadym E. Savanevych ${ }^{2} \ddagger$, \\ Olexandr B. Briukhovetskyi ${ }^{2}$ and Artem V. Pohorelov ${ }^{1}$ \\ ${ }^{1}$ Kharkiv National University of Radio Electronics, \\ 14 Nauki Ave, Kharkiv UA-61166, Ukraine \\ email: sergii.khlamov@gmail.com \\ ${ }^{2}$ Western Radio Technical Surveillance Center, National Space Agency of Ukraine, \\ Kosmonavtiv St, Mukachevo UA-89600, Ukraine \\ email: vadym@savanevych.com
}

\begin{abstract}
In this article we described CoLiTec software for full automated frames processing. CoLiTec software allows processing the Big Data of observation results as well as processing of data that is continuously formed during observation. The scope of solving tasks includes frames brightness equalization, moving objects detection, astrometry, photometry, etc. Along with the high efficiency of Big Data processing CoLiTec software also ensures high accuracy of data measurements. A comparative analysis of the functional characteristics and positional accuracy was performed between CoLiTec and Astrometrica software. The benefits of CoLiTec used with wide field and low quality frames were observed. The efficiency of the CoLiTec software was proved by about 700.000 observations and over 1.500 preliminary discoveries.
\end{abstract}

Keywords. Methods: analytical, data analysis, numerical, statistical; techniques: image processing; surveys; astrometry; comets: general, individual: ISON; minor planets, asteroids

\section{Introduction}

The problem of asteroids and comets hazard is very actual and important now. That is why the surveys with automatic results processing are necessary for the most effective observations. During last few years a new generation of high productive telescopes and observatories has been grown significantly. This leads to the problem of efficient scientific exploitation of massive data sets, that can be a ground for the new research (Vavilova, 2012). Big Data requires the new computational methods for high efficiency data processing. Based on these methods some new software should be developed.

So, for the most effective astronomical observations with using of all advantages of Big Data the software with automated processing is necessary. For example, the CoLiTec software (Savanevych, 2012) allows Big Data processing in real time with archiving the multiply goals during single processing run, http://www.neoastrosoft.com.

\section{CoLiTec software}

CoLiTec project covers all possible tasks for automated frames processing (Savanevych, 2015), (Vavilova, 2012). This includes frames brightness equalization, moving objects detection, astrometry, photometry, etc. The CoLiTec software has abilities for detecting very slow and very fast objects (Savanevych, 2015). The range of visible velocities of

$$
\begin{gathered}
\dagger \text { sergii.khlamov@gmail.com } \\
\ddagger \text { vadym@savanevych.com }
\end{gathered}
$$




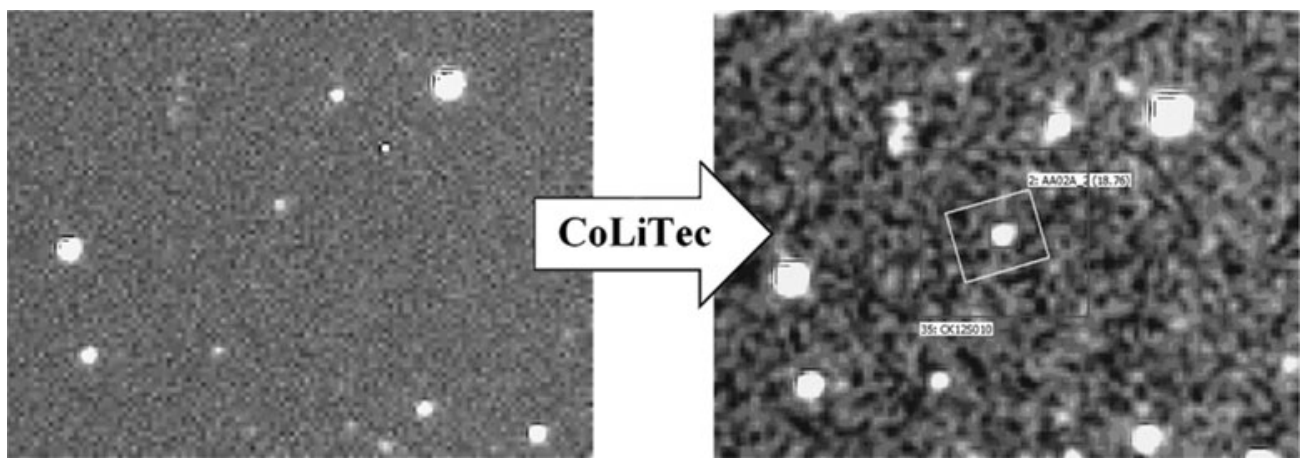

Figure 1. Discovery of C/2012 S1 (ISON) comet by CoLiTec software

detected objects is from 0.7 to 40.0 pix./frame. This is provided by the following features of CoLiTec software:

o Automatic detection of faint moving objects ( $\mathrm{SNR}>2.5)$;

o Working with very wide field of view (up to 10 degrees $^{2}$ );

o FrameSmooth software for brightness equalization;

o Auto calibration and cosmetic correction;

o Fully automatic robust algorithm of astrometric reduction;

o Automatic rejection of the worst observations;

o LookSky - processing results viewer with user-friendly GUI;

o Multi-threaded support for multi-cores systems and local network;

o Processing pipeline managed by OLDAS;

o CoLiTec Control Center (3C) with processing monitoring and logging.

These features substantiate CoLiTec software as effective complex solution at the different observatories in the world (Vavilova, 2012).

The especially significant part of CoLiTec software is the On-line Data Analysis System (OLDAS). It processes frames as soon as they are successfully uploaded on the server. This technique allows to greatly speed up processing and provides user immediate notification of emerging issues. OLDAS provides capabilities of the near real-time processing in the wide range of Big Data. For example, data sets of frames can be used for real-time full frame photometry. As a result of processing, the light curves of variable stars will be created and visualized on our server. Some other features are the estimation of the objects position (intraframe processing), moving objects detection (interframe processing) and confirmation of the most interesting objects at the night of their preliminary discovery.

Full reliability of the moving objects detection is retained up to the lower limit of SNR equal to 3 units in case of a minimum series consisting of four frames (Savanevych, 2015).

Moving objects detection on the series of CCD-frames is performed in two stages. The first one is the intraframe processing that allows estimating the objects positions at fixed times, cosmetic processing, frames alignment and brightness equalization.

The second stage of the moving objects detection is the interframe processing that allows detecting and estimating objects trajectories. Preliminary objects detection is based on the accumulation of statistics that are proportional to the signals energy along possible object's motion path. To perform such accumulation the multivalued transformation of the objects coordinates is used. It equals to the Hough space-time transformation (Savanevych, 2012). 

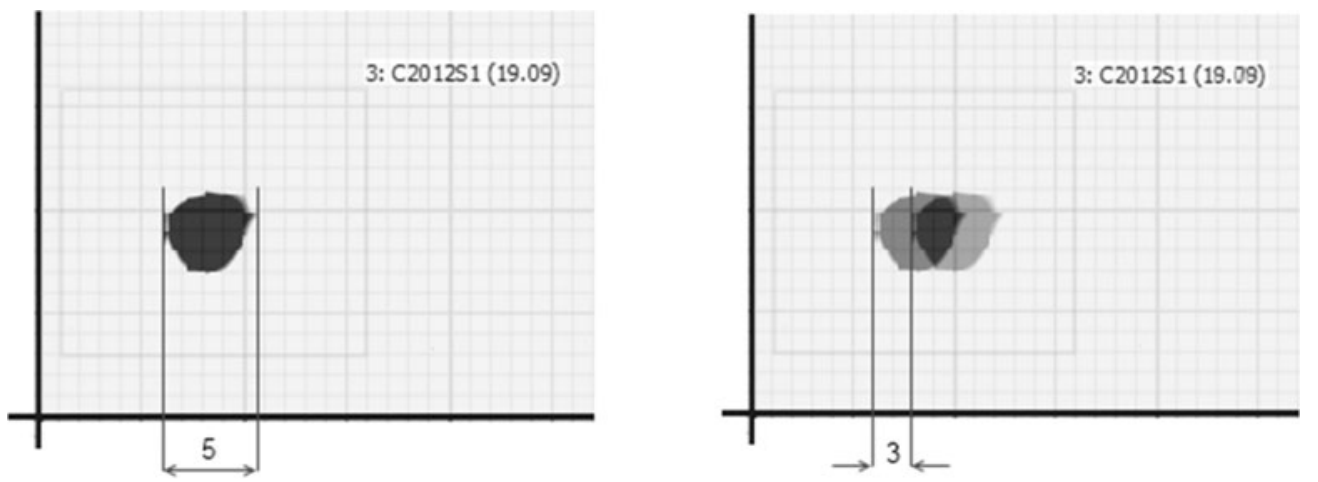

Figure 2. ISON comet: image size is 5 pixels, shift is 3 pixels

\section{Detection of the near-zero apparent motion}

The computational method for detection of the object's near-zero apparent motion on the series of CCD-frames (Khlamov, 2016) was implemented to CoLiTec software for automated asteroids and comets discoveries. It is based on checking the statistical significance of the factor of speed of the object's apparent motion on the series of measurements. This significance is checked by the two criteria. For coordinate-wise decision rules we use the Student's t-test and for XY-wise decision rule-F-test. To use all of synthesized decision rules the parameters of the object's apparent motion should be previously estimated as well as the interpolated coordinates on the frames should be determined.

After complex testing of the developed computational method for detection of the object's near-zero apparent motion it was embedded to the block of the interframe processing of the CoLiTec software.

Using the CoLiTec software and the proposed embedded computational method, the comet C/2012 S1 (ISON) (MPC, 2013)-long-period sungrazing comet was discovered, which at the time of discovery was the object with near-zero apparent motion (Fig. 1).

During 26 minutes of observation on series of 4 frames ISON comet's image was shifted on 3 pixels with own size of image of 5 pixels (Nevski, 2013) (Fig. 2).

\section{Comparative analysis between CoLiTec and Astrometrica software}

A comparative analysis of the functional characteristics and positional accuracy was performed between CoLiTec (Savanevych, 2015) and Astrometrica software (Raab, 2012). This comparison was performed on the same set of test CCD-frames.

19 series with 4 frames in each were used for comparative analysis of the accuracy. The preliminary analysis included processing of 36 series of frames. However, the frames of the rest series have not received reliable identification with used star catalog.

Also some frames with significant disruptions in the daily conduct and frames taken at very high winds were excluded. All the frames were made at the observatory ISONNM (MPC code - H15) (Elenin, 2013) from March 4, 2014 to March 30, 2014 by 0,4-m telescope SANTEL-400 with CCD-matrix FLI ML09000-65 (3056 x 3056 pixels, pixel size is 12 microns). Exposition time is $150 \mathrm{sec}$.

The positions of objects, which measurements are present in the archive MPCATOBS, were used. All measurements were processed by CoLiTec software (version 1.4.11.1 Autumn-2014) and Astrometrica (version 4.8.2.405). During researching etalon values of the positions of objects were received from HORIZONT service. 2002 measurements were analyzed. 
CoLiTec software made 253 measurements more than Astrometrica software. For these measurements Astrometrica software returned "Centroid=-1" (indication of impossible to guarantee reliable measurement of the position of object). Some reasons of this are attempts to measure the position of blurred objects or faint objects involved with a brighter star. More than half of them have $\mathrm{SNR}<3.5$.

The limits for reliable positional CCD-measurements using the CoLiTec software are wider than those of the Astrometrica software and the extension corresponds to the area of extremely low SNR. As a result, the objects that cannot be noticed by eyes are found. In case of low signal to noise ratios, the standard deviation of positional CCDmeasurements using the Astrometrica software is 30 - 50\% greater than that of the CoLiTec software.

The region of small SNR is valuable because everything new lies in it. At SNR $>6$, average deviations of CoLiTec and Astrometrica software are approximately the same in general case. CoLiTec software has the less frequency of critical values in deviations of both equatorial coordinates. So, the comparative analysis of the functional characteristics and positional accuracy showed the benefits of CoLiTec used with wide field and low quality frames (Savanevych, 2015).

\section{Conclusions}

During last years CoLiTec project has been continuously improving computational methods and principles for solving the problem of the efficient scientific exploitation of Big Data (Vavilova, 2012).

This allowed CoLiTec software (Savanevych (2015)) made over 1.500 preliminary discoveries of asteroids, including 5 NEO, 21 Trojan asteroids of Jupiter and 1 Centaur. It has been used in about 700000 observations, during which four comets (C/2010 X1 (Elenin), P/2011 NO1 (Elenin), C/2012 S1 (ISON), P/2013 V3 (Nevski)) were discovered.

The comparative analysis of the accuracy was performed between the CoLiTec and Astrometrica software. The analysis showed the benefits of the CoLiTec software used for astrometry and photometry of asteroids in relation with Astrometrica using (Savanevych, 2015).

\section{References}

Elenin, L., Savanevych, V., \& Bryukhovetskiy, A. 2013. Minor Planet Circulars, 82692, 1. Entrevue avec Vitali Nevski 2013. Codecouvreur de la comete ISON. Astronomie-Quebec, 2, 4 Khlamov, S. V., et al. 2016. Eastern-European Journal of Enterprise Technologies, 80, 41-48.

Minor Planet Center, COMET C/2012 S1 ISON 2012. Available at: http://www.minorplanetcenter.org/mpec/K12/K12S63.html

Raab, Herbert 2012. Astrophysics Source Code Library, record ascl:1203.012

Savanevych, V. E., et al. 2012. Space Science and Technology, 18, 39-46

Savanevych, V. E., et al. 2015. Monthly Notices of the RAS, 451, 3287-3298

Savanevych, V. E., et al. 2015. Kinematics and Physics of Celestial Bodies, 31, 302-313

Vavilova, I. B., et al. 2012. Kinematics and Physics of Celestial Bodies, 28, 85-102

Vavilova, I. B., et al. 2012. Baltic Astronomy, 21, 356-365 